\title{
A DSP-BASED ACTIVE CONTOUR MODEL
}

\author{
Juan Zapata \\ Depto. de Electrónica, Tecnología de Computadoras y Proyectos.Universidad Politécnica de Cartagena. \\ Campus Muralla del Mar s/n. Cartagena, Spain \\ Email: juan.zapata@upct.es \\ Ramón Ruiz \\ Depto. de Electrónica, Tecnología de Computadoras y Proyectos.Universidad Politécnica de Cartagena. \\ Campus Muralla del Mar s/n. Cartagena, Spain \\ Email: ramon.ruiz@upct.es
}

\begin{abstract}
Key words: Ultrasound image processing, DSP processors, active deformable contours.
Abstract: In this paper a DSP-based active contour model for tracking of the endocardium in a sequence of echocardiographic images is presented. If a contour is available in the first frame of a sequence, the contours in the subsequent frames are segmented. Deformable active contours is a technique that combine geometry, physics and approximation theory in order to solve problems of fundamental importance to medical image analysis; such as segmentation, representation and matching of shapes, and the tracking of objects in movement. The procedure has been developed on a DSP processor using its hardware features. The results are illustrated using a sequence of four-chambers apical echocardiographic images.
\end{abstract}

\section{INTRODUCTION}

In recent years, ultrasound image processing has attracted the attention of researchers in the study of diagnostic systems based on the analysis of images, mainly due to the non-ionizing nature of ultrasonic radiation and the consequent reduction of risks for the patient and the medical professional. In this respect, it can be confirmed that some aspects of techniques used in the analysis of images have been strongly influenced by the development of solutions to typical problems in this domain such as the recognition of areas with anatomical meaning in the image and nonrigid movement tracking.

Operations developed for image processing are computationally intensives, mainly due to the tremendous volume of optical information and the complex mathematical operations required. This aspect of computational demand becomes decisive when a real-time result is required. General purpose processors are not appropriate for real-time medical processing imaging applications because of technical tradeoffs in hardware design. General purpose processors are extremely capable when it comes to data manipulation but are inefficients for mathematical calculation. DSP (digital signal processing) processors work in an opposite way since they are efficient for mathematical calculation and less efficients in data manipulation (Lapsley et al., 1996). Digital signal process- ing is one of the most powerful technologies and has shown enormous growth and had an important technological impact in several areas, e.g. in telecommunications, medical imaging, radar and sonar, high fidelity music reproduction, to name just a few. DSP systems are perfectly capable of solving the problem of endocardium motion tracking in echocardiography images.

Numerous papers have considered the problem of automatic contour tracking. One approach is to use active contour models to track the boundaries of anatomic structures in medical images. This technique is a suitable alternative to classic edge-based segmentation techniques, and combines the detection of grey level transitions within the image together with the need to obtain a closed contour. This task involves both hardware and software aspects. In fact, while purely hardware solutions have proved to be inefficient and unsuitable for complex algorithms, a purely software solution based on a PC does not offer a large bandwidth in terms of MOPS and system throughput (Geminami et al., 1999). Therefore, an integrated hardware/software platform based on a DSP processor located in a host PC was chosen as environment to develop active contour algorithms. Due to its full programmability, high performance and novel architecture, the TMS320C6701 is one of the most suitables devices for researching and testing new digital signal processing algorithms. 


\section{A CONTOUR TRACKING ALGORITHM}

Geometrically, a snake or deformable active contour is a spline with a parametric representation $\mathbf{v}(s)=(x(s), y(s))$ embedded in the image plane $(x, y \in R)$, where $x$ and $y$ are the coordinate function and $s \in[0,1]$ is the parametric domain (Kass et al., 1988). Energetically, the active contour is subordinated to two types of energy that dictate its behaviour. Equation (1) can be viewed as a representation of the energy of the contour and the final shape corresponds to the minimum of this energy.

$$
E_{\text {snake }}^{*}=\int_{0}^{1} E_{\text {int }}(\mathbf{v}(s))+E_{\text {ext }}(\mathbf{v}(s))
$$

The first term of the functional is the internal deformation energy. This internal energy (2) consists of the first and second derivatives of $\mathbf{v}(s)$ in relation to $s$ and characterizes the deformation of a stretchy, flexible contour. Two parametric functions, $\omega_{1}(s)$ and $\omega_{2}(s)$, dictate the simulated physical characteristics of the tension and rigidity of the contour, respectively.

$$
\begin{aligned}
& \int_{0}^{1} E_{i n t}(\mathbf{v}(s)) d s= \\
& \int_{0}^{1} \omega_{1}(s)\left|\frac{\partial \mathbf{v}(s)}{\partial s}\right|+\omega_{2}(s)\left|\frac{\partial^{2} \mathbf{v}(s)}{\partial s}\right|^{2} d s
\end{aligned}
$$

The second term, the external energy, also termed the image energy, couples the snake to the image. Traditionally,

$$
\int_{0}^{1} E_{\text {ext }}(\mathbf{v}(s)) d s=\int_{0}^{1} P(\mathbf{v}(s))+E_{\text {con }}(\mathbf{v}(s)) d s
$$

where $P(\mathbf{v}(s))$ denotes a scalar potential function defined on the image plane. This scalar function can be designed so that local minima coincide with intensity extrema, edges or other image features of interest. The second term of the equation (3), $E_{c o n}(\mathbf{v}(s))$, is the energy consequence of external constraints. These asserted constraints can benefit certain zones or local characteristics of the image. Therefore, during the minimization procedure, the contour can be favoured to reach these zones.

There are two key difficulties with the original technique. First, the procedure requires that the initial contour lies near the feature of interest and relies on an inherent force to move the contour towards the edges. Cohen (Cohen, 1991) decided to include of a force to oblige the active contour to expand and to contract in the absence of other type of forces in order to solve some of the problems encountered in the original model, where main problem was that a snake which is not close enough to the edges or interest features is not attracted by them, and so the object contour is reached very slowly or converges to a straight line. The model developed by Cohen allows a behaviour like a balloon since the curve is driven by an internal pressure force. In summary, our model proposes to use this pressure force in order to allow a faster approximation of an active contour when it is not near the real contours of the object to segment. Cohen formulates the balloon model in the following form: $F=k_{1} \vec{n}(s)-k \frac{\nabla P}{\|\nabla P\|}$, where $\vec{n}(s)$ is a normal unitary vector to the curve at point $\mathbf{v}(s), k_{1}$ is the force amplitude and $k$ a scale factor and $\mathrm{P}$ is the image potential. Other methods have been proposed to solve the problem of initialization (Leroy et al., 1996; Cohen and Cohen, 1993). The main idea, common in others techniques too, is to increment the capture range of the external forces and to guide the initial contour towards the desired contour.

A second problem appears when the active contour cannot progress in the concavities of the object (Davatzikos and Prince, 1995). There is no successfull solution to this problem, although several forces have been proposed by researchers (Cohen, 1991; Davatzikos and Prince, 1995; Davatzikos and Prince, 1994; Prince and $\mathrm{Xu}, 1996)$. However, although many of these proposed methods solve theproblem they given rise to new difficulties. For example, pressure forces must be initialized to push out or push in, a condition that involves careful initialization. $\mathrm{Xu}$ and Prince designed a new external force called gradient vector flow force or GVF force (Xu and Prince, 1997; $\mathrm{Xu}$ and Prince, 1998); which is intended to solve the aforementioned problems. The gradient vector flow field is a vectorial field which is derived from the image through the minimization of an energy functional. This external GVF force is computed as a diffusion of the gradient vectors of a gray-level or binary edge map derived from the image. The GVF field can be found by solving the following Euler equations, where $\nabla^{2}$ is the Laplacian operator:

$$
\begin{aligned}
& \mu \nabla^{2} u-\left(u-f_{x}\right)\left(f_{x}^{2}+f_{y}^{2}\right)=0 \\
& \mu \nabla^{2} v-\left(v-f_{x}\right)\left(f_{y}^{2}+f_{y}^{2}\right)=0
\end{aligned}
$$

Equations (4) and (5) can be rewritten as follows:

$$
\begin{aligned}
& u_{t}(x, y, t)= \\
& \mu \nabla^{2} u(x, y, t)-b(x, y) u(x, y, t)+c^{1}(x, y) \\
& v_{t}(x, y, t)= \\
& \mu \nabla^{2} v(x, y, t)-b(x, y) v(x, y, t)+c^{2}(x, y)
\end{aligned}
$$

where:

$$
\begin{aligned}
b(x, y) & =f_{x}(x, y)^{2}+f_{y}(x, y)^{2} \\
c^{1}(x, y) & =b(x, y) f_{x}(x, y) \\
c^{2}(x, y) & =b(x, y) f_{y}(x, y)
\end{aligned}
$$

We have introduced a hybrid energy term (Sánchez et al., 2000), which is an adaptive combination of both 
terms, GVF and balloon energy. In this way, if there is no GVF field, then the balloon force acts on the active contour, although its influence over the active contour decreases as the GVF force increases in magnitude. The superposition of effects get through the balloon force and the GVF force is ruled by a user parameter. The optimum value of this parameter is obtained when the influence of the pressure force over the active contour is null.

\section{HARDWARE OVERVIEW}

\subsection{TMS320C60 EVM evaluation board}

The choice of DSP-based hardware permits the development of a powerful and highly flexible system. Due to its full programmability and high performance, the TMS320C6701 processor mounted on the TMS320C60 EVM is a suitable device for researching and testing DSP algorithms. In the work described in this paper, a personal computer was equipped with the Code Composer Studio development environment which helps to construct and debug embedded real-time DSP applications. It provides tools for configuring, building, debugging, tracing and analyzing programs. Texas Instruments DSP's provide on-chip emulation support that enables Code Composer Studio to control program execution and monitor real-time program activity. The heart of the TMS320C60 EVM evaluation board is the Texas Instruments TMS320C6701 processor. The C6701 is based on a VLIW-like architecture which allows it to execute up to eight RISC-like instructions per clock cycle.

The two data paths of the C6701 extend the functionality of the data paths of the C6201 with support for 64-bit data and IEEE-754 32-bit single-precision and 64-bit double-precision floating-point arithmetic. Each data path includes a set of four execution units, a general-purpose register file, and paths for moving data between memory and registers. The four execution units in each data path comprise two ALUs, a multiplier and an adder/subtractor which is used for address generation. The ALUs support both integer and floating point operations, and the multipliers can perform both $16 \times 16$-bit and 32x32-bit integer multiplies and 32-bit and 64-bit floating point multiplies. The two register files each contain sixteen 32-bit general-purpose registers. To support 64-bit floating point arithmetic, pairs of adjacent registers can be used to hold 64-bit data. In addition to the operations supported by the C6201, the C6701 offers support for floating-point reciprocal and recip- rocal square root estimation, and for converting data between fixed- and floating-point formats.

\subsection{Implementation}

Although the code running on the DSP processor can be written in $\mathrm{C}$ because the compiler generates an quasi-efficient code, the performance of the application can be maximized by using compiler options, intrinsic instructions, and assembly code transformation. In fact, this previous strategy was used in our code.

The computational cost of the active contour algorithm constitutes most the computational cost of the entire procedure. Therefore, the algorithm is implemented in assembly language on the four execution units in each data path and executed in parallel in order to utilize all the hardware resources of DSP and exploit all the capabilities of the architecture VLIW and software pipelining. Because most of the millions of instructions per second (MIPS) in DSP applications occur in tight loops, it is important for the application to make maximal use of all the hardware resources in important loops. Fortunately, loops have more inherent parallelism than non-looping code because multiple iterations of the same code are executed with limited dependency between each iteration.

To maximize the efficiency of the code, the application schedules as many instruction as possible in parallel. For this, the relationships, or dependencies, between instructions were determined. Figure 1 shows the assembly instructions for floating-point active contour and allocated resources. The symbol || defines which instruction is being executed in parallel, while the symbol @ defines which iteration of the loop is executing each cycle. For example, the rightmost column shows that on during given cycle inside the loop, the ADDSP instruction is adding data for iteration $\mathrm{n}$, the MPYSP instruction is multiplying data for iteration n+2 (@@), and so on. No value can live in a register for more than the number of cycles in the loop. Otherwise, iteration $n+1$ writes into register before iteration $\mathrm{n}$ has read that register. The live-too-long problem means that no loop variable can live longer than the iteration interval, because a child would then read the parent value for the next iteration. A simple solution is to break up the lifetime of a variable by inserting move (MV) instructions in order to break up the path of the variables into smaller pieces and can live for minimum iteration interval. One way to accomplish if-then-else in assembly code is by means of conditional instructions in one of the five general-purpose registers. Conditional instructions can handle both the true and false cases of ifthen-else statement. Branching is one way, although, because each branch has five delay slots, this method requires additional cycles. Furthermore, branching 
within the loop makes software pipelining almost impossible. Using conditional instructions eliminates the need to branch to the appropriate piece of code after checking the condition. For this, instructions can be used on the zero and nonzero values of a condition register. This method also allows software pipeline the loop and achieve much better performance.

\section{RESULTS AND DISCUSSION}

The high speed contour tracking procedure was tested on sequences of cardiovascular images. The video was captured with a resolution of $512 \times 512$ pixels, 8 bits per pixel, 25 frames per second.

The procedure, designed to track the endocardial walls, used a hybrid snake to segment the first frame of the ultrasound sequence (see Figure 4). In the next frames, the active contour is located under the GVF force derived from a GVF field of reduced diffusion. A GVF field of reduced diffusion cannot close all the discontinuities, and so if the pressure force is found in any place of the image, it may deform the contour further of endocardial boundaries or invade other chambers. In this case, it is necessary to deactivate the force although there is no GVF field. In this way, the GVF snake (very close to its real contour) is only influenced by the rest of the concerned forces and deformations are not produced. Our experiments are based on tracking each chamber in different echocardiographic sequences. In summary, Figure 3 shows the echocardiographic sequence for the left ventricle (three frames are shown), with the active contours achieved in the first column and drawn by a specialist in the second column. The similarity is about a $94.5 \%$ for the first and second frame and a $94.7 \%$ for the third frame. Similar results were achieved for different frames of the sequence and for the others chambers.

The software was implemented and configured to process instructions in parallel. To be more exact, loop kernel can be executed in 7 cycles only. Every execution packet involves at least two instructions executing in parallel, although there are packets with five instruction in parallel and an execute packet with seven instruction in parallel. Therefore, if we suppose our snake has 80 snaxels then the energy of a neighboring point is calculated in each iteration (7 cycles). Each snaxel has 9 neighbours and, therefore, $80 \times 9 \times 7=5040$ cycles are needed for the active contour in a frame. In a complete sequence, in one second, $5040 \times 25=125.800$ cycles are needed and the DSP load is just $0.1 \%(125 K / 160 M \times 100)$. Given these circumstances, the implementation can be considered very satisfactory for the DSP adopted.

We have presented a high-speed contour tracking

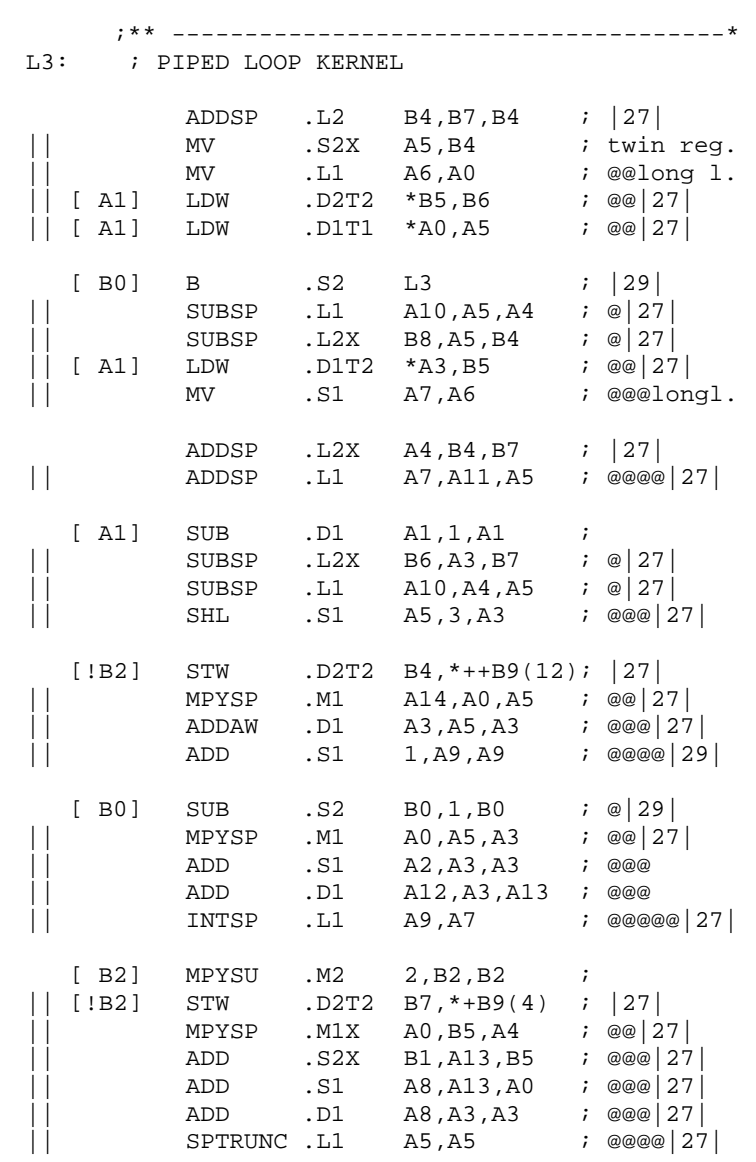

Figure 1: Loop kernel for the active energy algorithm 


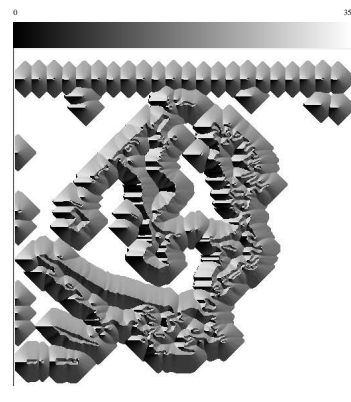

(a) GVF field of reduced diffusion

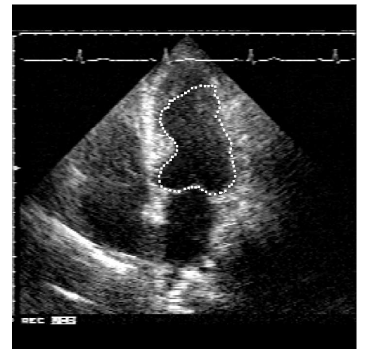

(b) Hybrid snake

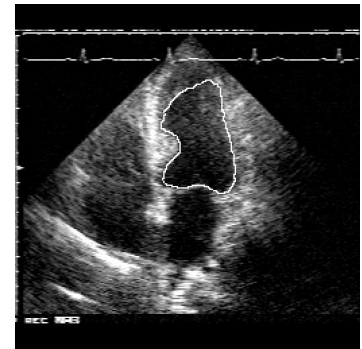

(c) Boundary proposed by a expert human

Figure 2: Left ventricle boundary delimitation

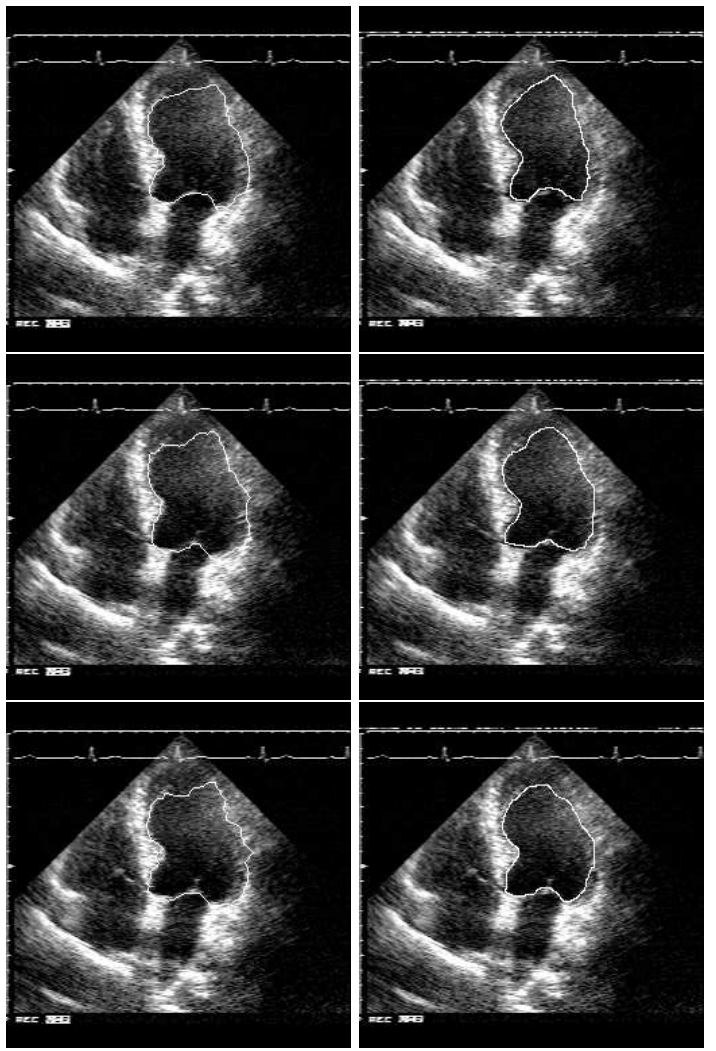

Figure 3: Tracking of the left ventricle in a sequence of ultrasound images. 
procedure where a snake is used to contour the endocardium in a sequence of echocadiographic images. Our preliminary results are encouraging. The main effort consists in the development of a user interface. This interface must communicate the host with the application running on the DSP, must initialize the active contour and set up the tuning parameters. Our system should be tested with other echographic images to measure its real performance and tuning capacity.

\section{REFERENCES}

Cohen, L. (1991). On Active Contour Models and Balloons. Computer Vision, Graphics and Image Proccessing, 53(2):211-218.

Cohen, L. and Cohen, I. (1993). Finite-Element Methods for Active Contour Models and Balloons for 2-D and 3-D Images. IEEE Trans. Pattern Analysis and Machine Intelligence, 15(11):1131-1147.

Davatzikos, C. and Prince, J. L. (1994). Convexity Analysis of Active Contour Models. In Proc. Conf. on Info. Sci. and Sys., pages 581-587.

Davatzikos, C. and Prince, J. L. (1995). An Active Contour Model for Mapping the Cortex. IEEE Trans. on Medical Imaging, 14(1):65-80.

Geminami, V., Provvedi, S., Demi, M., Paterni, M., and Benassi, A. (1999). A DSP-Based Real Time Contour Tracking System. IEEE.

Kass, M., Witkin, A., and Terzopoulos, D. (1988). Snakes: Active Contour Models. International Journal of Computer Vision, 1(4):321-331.

Lapsley, P., Bier, J., Shoham, A., and Lee, E. (1996). DSP Processor Fundamentals: Architectures and Features. IEEE Press.

Leroy, B., Herlin, I., and Cohen, L. D. (1996). Multiresolution Algortihms for Active Contour Model. In 12th International Conference on Analysis and Optimization of System, pages 58-65.

Prince, J. and Xu, C. (1996). A New External Force Model for Snakes. In 1996 Image and Multidimensional Signal Processing Workshop, pages 30-31.

Sánchez, P. J., Zapata, J., and Ruiz, R. (2000). An active contour model algorithm for tracking endocardiac boundaries in echocardiographic sequences. Criticals Reviews in Biomedical Engineering.

Xu, C. and Prince, J. (1997). Gradient Vector Flow: A New External Force for Snakes. In IEEE Proc. Conf. on Comp. Vis. Patt. Recog. (CVPR), pages 66-71.

$\mathrm{Xu}, \mathrm{C}$. and Prince, J. (1998). Snakes, Shapes, and Gradient Vector Flow. IEEE Transactions on Image Processing, pages 359-369. March. 